\title{
Complex coronary arterial fistulas with mediastinal hemangioma: a case report and review of the literature
}

\author{
Yutian Sun ${ }^{1}$, Nan Wang ${ }^{2}$, Hongkun Shi ${ }^{2}$ and Dianbo Cao ${ }^{2 *}$ \\ ${ }^{1}$ Pharmaceutical Department, China-Japan Union Hospital, Jilin University, Changchun 130033, China \\ ${ }^{2}$ Department of Radiology. The First Hospital of JiLin University, Chang Chun 130021, China
}

\begin{abstract}
Coronary arterial fistula is a connection between one or more of the coronary arteries and a cardiac chamber or great vessel, having bypassed the myocardial capillary bed. We reported such a patient with chest tightness, head dizziness and headache. Coronary angiography showed mediastinal tortuous vessels from the branch of left circumflex coronary artery, but unable to define its termination. Coronary CT angiography demonstrated a large tortuous vessel with the communication between the branch of left circumflex artery and the branch of descending thoracic aorta. Meanwhile, these tortuous, dilated vessels were ascertained to have multiple microcommunications with right pulmonary trunk owing to its earlier shadow. The patient refused any intervention, and his condition remained well on the follow up of 12 months.
\end{abstract}

\section{Case report}

A 56-year-old man with a history of renal calculus 3 years before was admitted to our hospital, complaining of chest tightness under the xiphoid, head dizziness and headache three days previously. These symptoms were not associated with exertional exercise and posture. After oral quick-acting heart treatment pill, the patient's symptoms did not significantly improve. The patient's condition was well without clinical evidence of congestive heart failure (distension of jugular veins and bilateral basal crackles). Blood chemistries, including coagulation studies and complete blood cell count, as well as cardiac enzymes and lipid profiles, were all within the normal limits. Coronary angiography demonstrated no significant narrowing of three major coronary arteries. However, a dilated, tortuous vessel originating from the branch of left circumflex coronary artery and going into mediastinum was noticed associated with slowly emptying, but unable to define its final destination (Figures 1 and 2). He subsequently underwent an ECG-gated contrast-enhanced coronary CT angiography using a dual-source 128 slice multidetector CT. This demonstrated a large tortuous, dilated vessel with the communication between the branch of left circumflex artery running along the dome of the left atrium and the branch of descending thoracic aorta in the mediastinal region (Figure 3). These tortuous, dilated vessels were ascertained to have multiple micro-communications with right pulmonary trunk owing to its earlier shadow in comparison to left pulmonary trunk (Figures 4 and 5). Finally, complex coronary artery fistulas with multiple sites of origin and drainage were diagnosed. Treatment modalities including surgical ligation, transcatheter closure and regular follow-up were all proposed, and he refused any intervention. On the follow up of 12 months, his condition remained stable.

\section{Discussion}

A coronary arterial fistula (also known as coronary arteriovenous malformation) is a connection between one or more of the coronary arteries and a cardiac chamber or great vessel, having bypassed the myocardial capillary bed. It is report that $0.1 \%-0.2 \%$ of all patients who undergo selective coronary angiography are diagnosed with CAF [1]. The majority of the fistulas have a congenital origin accounting for approximately $14 \%$ of all congenital cardiac defect but may also be acquired as a complication following trauma or invasive cardiac procedures (pacemaker, endomyocardial biopsy, coronary angiography, septal myomectomy). Any of the three major coronary arteries can be the feeding artery for the coronary fistula: right coronary artery (37\%), left coronary artery (33\%), left anterior descending artery $(26 \%)$, and left circumflex artery (4\%). One-third of these cases was

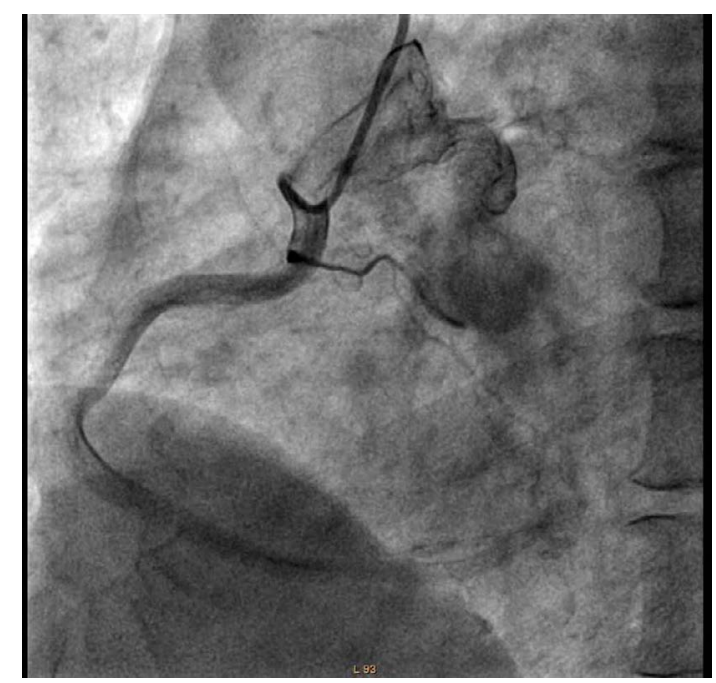

Figure 1. A dilated, tortuous vessel originating from the branch of left circumflex coronary artery.

${ }^{\star}$ Correspondence to: Dianbo Cao, Department of Radiology, The First Hospital of Jilin university, 71 XinMin, Zhu Street, Chang Chun, China

Received: May 20, 2018; Accepted: May 29, 2018; Published: June 03, 2018 


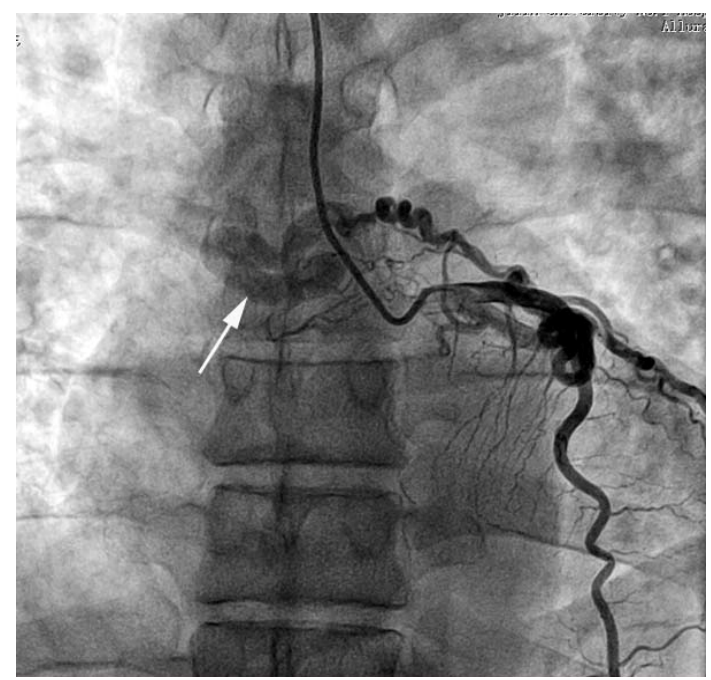

Figure 2. A dilated, tortuous vessel going into mediastinum was noticed associated with slowly emptying, but unable to define its final destination.

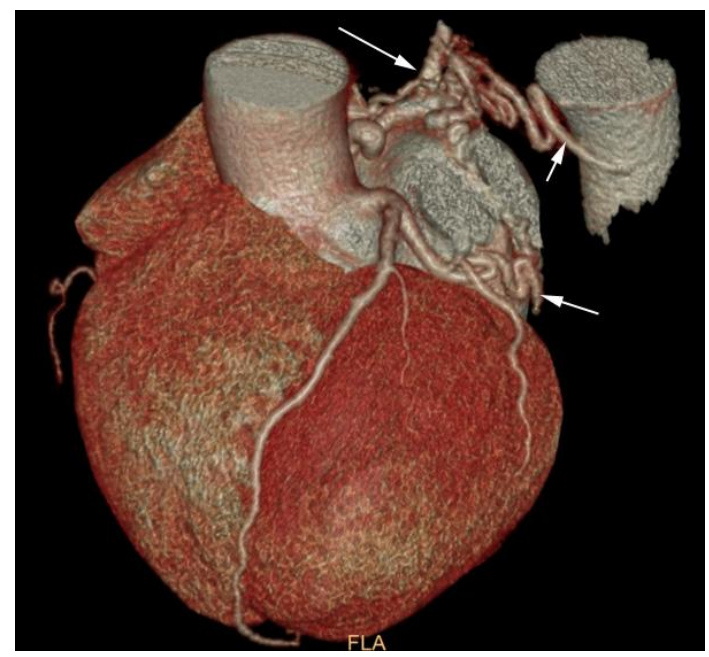

Figure 3. Demonstrated a large tortuous, dilated vessel with the communication between the branch of left circumflex artery running along the dome of the left atrium and the branch of descending thoracic aorta in the mediastinal region.

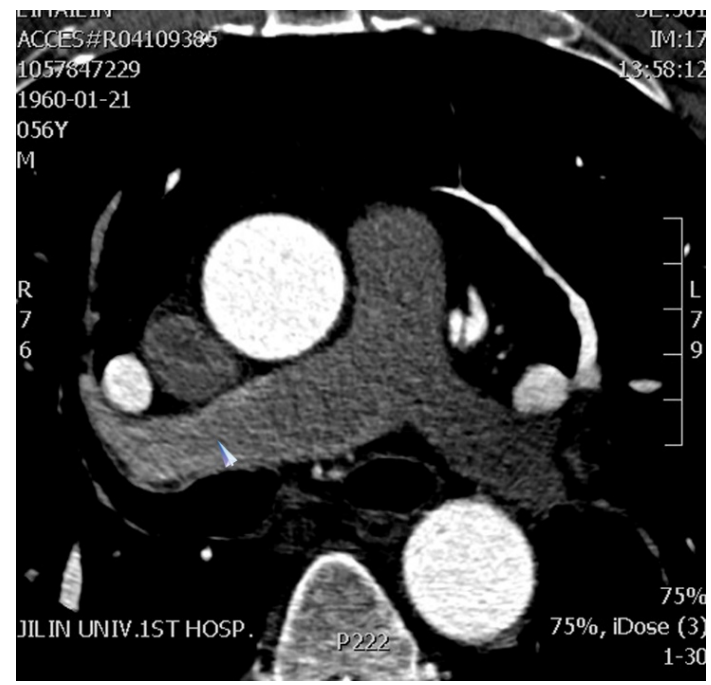

Figure 4. These tortuous, dilated vessels were ascertained to have multiple microcommunications with right pulmonary trunk owing to its earlier shadow in comparison to left pulmonary trunk.

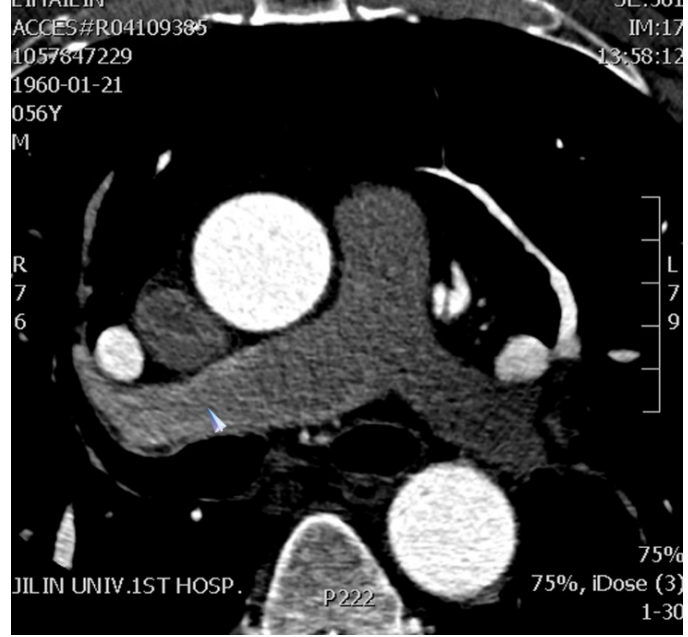

Figure 5. Finally, complex coronary artery fistulas with multiple sites of origin and drainage were diagnosed. Treatment modalities including surgical ligation, transcatheter closure and regular follow-up were all proposed, and he refused any intervention.

associated with aneurysm [2]. Feeding artery from the branch of aorta is rarely reported in the literature, as described in our patient. Depending on the artery and point of origin, the tortuosity and dilatation of the fistula will markedly vary. CAFs that branch from a main coronary artery at a proximal location are more dilated when compared to other distally located CAFs. For example, fistulas that originate proximally and drain into the right atrium are more dilated but less tortuous. The communication site of CAFs could include: right ventricle accounting for $41 \%$, right atrium $26 \%$, left atrium $5 \%$, left ventricle $3 \%$, coronary sinus $7 \%$, superior vena cava $1 \%$, pulmonary artery $17 \%$ or the pulmonary vein [3]. It is important to alert that there may be multiple feeding arteries to a single CAF drainage point or there may be multiple drainage sites, as mentioned in this case. Multiple fistulas can be seen in $11 \%-16 \%$ of cases [4].

Based on several studies, symptoms are present in 19-63\% of patients with CAFs, with the majority occurring after 18 years of age. Large fistula leads to preferential blood flow from coronary circulation to low-pressure pulmonary circulation, resulting in pulmonary hypertension and coronary-steal-related chronic myocardial ischemia. Presented symptoms include continuous heart murmur, dyspnea, orthopnea, right ventricular dysfunction/failure, fatigue, chest pain, endocarditis, stroke, arrhythmias, myocardial ischemia/infarction due to coronary steal, pericardial effusion or sudden death, cardiac failure, pulmonary hypertension, thrombosis, rupture, and aneurysm. Thrombosis within the fistula is rare but may cause acute myocardial infarction, and atrial and ventricular arrhythmias. Spontaneous rupture of the aneurysmal fistula causing haemopericardium has also been documented $[5,6]$. These symptoms are difficulty to improve when administering vasodilator drug, as this patient described.

Due to the asymptomatic nature of most CAFs, many are incidentally detected during routine examinations or investigating other reasons. Presently, various imaging modalities are available for evaluating CAFs. Conventional coronary angiography (CAG), as the gold standard for identifying CAFs, may not sufficient for defining the complex structural anatomy and multiple fistulas arising from different segments of the coronary arteries and coronary sinuses. Temporal and spatial resolution of MR angiography and echocardiography was inferior to that of CAG. Coronary CT angiography is relatively new imaging modality that has been used for non-invasive coronary artery imaging since 2000. Prior to this earlier system, produced images 
were of poor quality due to limitations with spatial and temporal resolution and image noise. ECG-gated dual tube 128-slice MDCT can produce high quality images with the ECG-gated image reconstruction algorithms allowing phase-correlated image data sets. Coronary CTA can clearly delineate cardiac chambers, coronary arteries, coronary veins and corresponding thoracic arota. Now, its clinical significance in CAFs diagnostics has been identified due to its ability to provide detailed images, such as CAFs, possible aneurysms, and adjacent anatomical structures [7]. To date, complex coronary artery fistulas similar to our case have rarely reported, which renders vital value of coronary CTA.

Presently, no consensus is reached on the optional management of CAFs. CAFs are conservatively treated with imaging follow up for asymptomatic patients. While rare, there have been cases of spontaneous closure of CAFs without surgical ligation or catheter repair in few patients reported. Among them, most spontaneous closures occur in children diagnosed with a CAF prior to 2 years of age and these fistulas almost drain into the right ventricle [8]. The main indications for closure of the fistula include the presence of a large or increasing left-to-right shunt, left ventricular volume overload, myocardial ischemia, left ventricular dysfunction, congestive cardiac failure and for prevention of endocarditis/endarteritis. The treatment options may include surgery or catheter closure. Surgery involves internal closure of the fistula within the receiving chamber or vessel whenever feasible, but when the fistula is associated with a large aneurysm of the feeding artery, it may need to be ligated from within the aneurysm. Many retrospective reviews conclude that complications involved in surgical correction outweigh the risk of CAFs itself $[9,10]$. So, large shunts are present but do not cause pathological problems or symptoms, treatment options remain controversial. Catheter closure of the fistulas in those eligible patients is increasingly considered to be an effective and safe alternative to surgery $[11,12]$. The technique of catheter closure further allows arterial feeding vessels to be discovered by selective coronary angiography at the end of the procedure and if such multiple feeding vessels are noted, these can also be occluded simultaneously. Therefore, some centers perform closure even in asymptomatic patients to avoid future morbidity and mortality by transcatheter technique. Based on these opinions above, our management conforms with the patient's consent.

\section{Conclusion}

In complex CAFs with multiple sites of origin and drainage may be challenging to diagnose. Coronary CTA is vital in precise characterization of the fistula anatomy and had better be performed in all patients with a diagnosis of CAFs made via conventional angiography.

\section{References}

1. Said SA, EI Gamal MI, Werf T Van der (1997) Coronary arteriovenous fistulas: collective review and management of six new cases-changing etiology, presentation, and treatment strategy. Clin Cardiol 20: 748-752. [Crossref]

2. Sharma UM, Aslam AF, Tak T (2013) Diagnosis of coronary artery fistulas: clinical aspects and brief review of the literature. Int J Angiol 22: 189-192. [Crossref]

3. Wilcox WD, Neal MJ, Alpert BS, Taylor AB, Dooley KJ (1986) Localized occurrence of congenital coronary artery fistula in the southeast United States. Am J Cardiol 57: 361-363.

4. Dodge-Khatami A, Mavroudis C, Backer CL (2000) Congenital heart surgery nomenclature and database project: anomalies of the coronary arteries. Ann Thorac Surg 69: S270-s297. [Crossref]

5. Ramo OJ, Totterman KJ, Harjula AL (1994) Thrombosed coronary artery fistula as a cause of paroxysmal atrial filbrillation and ventricular arrhythmia. Cardiovasc Surg 2: 720-722. [Crossref]

6. Bauer HH, Allmendinger PD, Flaherty J, Owlia D, Rossi MA, et al. (1996) Congenital coronary arteriovenous fistula: spontaneous rupture and cardiac tamponade. Ann Thorac Surg 62: 1521-1523. [Crossref]

7. Min liu, Qing Hou, Xiaojuan Guo, Shuangkun Wang, Zhanhong Ma (2014) Dualsource CT coronary angiographic evaluation of coronary artery fistulas. Exp Ther Med 7: 1155-1159. [Crossref]

8. Schleich JM, Rey C, Gewilling M, Bozio A (2001) Spontaneous closure of congenital coronary artery fistulas. Heart 85: e6. [Crossref]

9. Wang S, Wu Q, Hu S, Sun L, Song Y, Lu F (2001) Surgical treatment of 52 patients with congenital coronary artery fistulas. Chin Med J 114: 752-755. [Crossref]

10. Kamiya H, Yasuda T, Nagamine H, Sakakibara N, Nishida S, et al. (2002) Surgical treatment of congenital coronary artery fistulas: 27 years' experience and a review of the literature. J Card Surg 17: 173-177. [Crossref]

11. Mottin B, Baruteau A, Boudjemline Y, Guérin P (2016) Transcatheter: A French multicenter study. Catheter Cardiovasc Interv 87: 411-418.

12. Ilkay E, Celebi OO, Kacmaz F, Ozeke O (2015) Percutaneous closure of coronary artery fistula: long-term follow-up results. Postepy Kardiol Interwencyjnej 11: 318 322. [Crossref]

Copyright: (C2018 Sun Y. This is an open-access article distributed under the terms of the Creative Commons Attribution License, which permits unrestricted use, distribution, and reproduction in any medium, provided the original author and source are credited. 